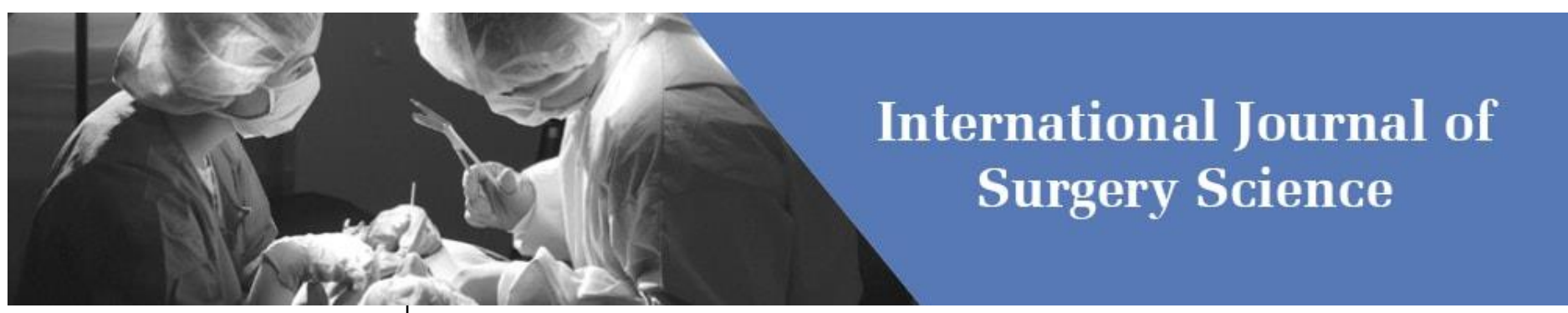

E-ISSN: 2616-3470

P-ISSN: 2616-3462

(C) Surgery Science

www.surgeryscience.com

$2020 ; 4(2): 218-222$

Received: 08-02-2020

Accepted: 10-03-2020

Dr. Ketan Vagholkar

Professor, Department of Surgery.

DY Patil University School of

Medicine Navi Mumbai,

Maharashtra, India
Corresponding Author: Dr. Ketan Vagholkar Professor, Department of Surgery.

DY Patil University School of

Medicine Navi Mumbai,

Maharashtra, India

\section{Complications of splenectomy}

\section{Dr. Ketan Vagholkar}

DOI: https://doi.org/10.33545/surgery.2020.v4.i2d.420

\section{Abstract}

Spleen is an important organ of the reticuloendothelial system. It plays a crucial role in the immunological system of the body. Understanding the consequences and diagnosis of hyposlenic and asplenic states is essential. Splenectomy is performed for a variety of indications ranging from haematological conditions to trauma. Complications of splenectomy include surgical as well as immunological. Overwhelming post splenectomy infection is one of the most dreaded complication with high mortality. The physiological basis of immunological function of the spleen, hyposplenism and complications of splenectomy are presented in this paper.

Keywords: Post splenectomy, complications, hyposplenia, OPSI

\section{Introduction}

Spleen is a very important constituent organ of the reticuloendothelial system. The organ is crucial in regulating immune homeostasis through its ability to link innate and adoptive immunity in the process of protecting against infection. Hyposplenism is impairment of splenic function. It is usually acquired and caused by severe haematological and immunological disorders. Asplenia refers to the absence of the spleen which is rarely congenital but predominantly post-surgical (splenectomy). The most important complication of asplenic state is infectious complications ${ }^{[1]}$. These infections have high mortality. In addition to infectious complications, splenectomy can lead to a series of other complications.

\section{Structure of the spleen}

The spleen has three compartments, red pulp, white pulp and the marginal zone ${ }^{[1]}$. The red pulp is a spongy structure filled with blood flowing through the sinuses and cords. The white pulp srrounds the central arteriole which arises from the splenic artery. The $\mathrm{T}$ cells surround the central arteriole as well as the B cell follicle. This thin layer is surrounded by an outer dark zone, the mantle zone containing proliferating small B lymphocytes. The light central zone is the germinal centre. The marginal zone containing memory B cells is between the red and white pulp and is in direct contact with the perifollicular area wherein macrophages and fibroblasts position for mucosal cell adhesion molecules.

\section{Functions of the spleen}

The primary function of the spleen is phagocytic filtration. This involves removal of senescent and damaged red blood cells by the process of culling and removal of particles or inclusions from the cytoplasm of erythrocytes by pitting. Other functions include removal of blood borne organisms, production of antibodies and as a blood reservoir. Blood enters the splenic cords of the red pulp and then passes through the fenestrated epithelial borders entering into the venous sinuses. The flow then slows down which enables removal of defective RBC's and bacteria by the macrophages. Some bacteria are recognized directly while others need opsonisation by complement components, properdin or tuftsin. Once opsonized, phagocytosis becomes faster and easier. The opsonized bacteria are removed efficiently by the macrophages. Poorly opsonized bacteria are the capsulated ones especially Strep. Pneumonia. These are then cleared by the spleen by way of IgM memory B cells in the marginal zone of the spleen. Memory B cells are a functionally important entity ${ }^{[1]}$. They are of two varieties switched B cells and IgM memory B cells. Switched memory B cells produce high affinity antibodies and are protective against reinfection. IgM memory cells need the spleen for survival and production. 
These produce natural antibodies. The concentration of $\operatorname{IgM}$ memory cells is less in children less than two years of age due to marginal zone immaturity. Low concentrations are also seen in other conditions like, common variable immunodeficiency, splenectomised patients, congenital asplenia, hyposplenism and elderly patients. These patients therefore have increased susceptibility to infections by encapsulated bacteria.

\section{Diagnosis of splenic dysfunction}

Diagnosis of splenic dysfunction is generally based on assessment of the splenic filtering function by radioisotope methods or quantification of erythrocyte morphological abnormalities [2]. Radioisotope methods enable a morphofunctional assessment of the spleen by injection, uptake and clearance of particulate substances or radiolabelled tracers. However their use in clinical practice is limited by high costs and technical difficulties.

As the tests for detecting morphological changes in the erythrocytes are inexpensive and less invasive than radiolabelling, they are more suitable for clinical practice. The detection of Howell Jolly bodies is a useful method of screening for asplenia. Erythrocytes bearing membrane pits, visible on phase interference microscopy were detected in premature neonates and adults who had undergone splenectomy ${ }^{[3]}$. One of the functions of the spleen is to remove pits from circulating erythrocytes by pitting $[4,5,6]$. Therefore asplenic and hypo splenic states can lead to an increased number of circulating pitted erythrocytes.

Pitted erythrocytes correlate well with the splenic volume and is the gold standard for assessment of splenic dysfunction. The inverse correlation seen between pitted erythrocytes and IgM memory cells in splenectomised and hyposplenic patients indicate that the impairment of the filtering function as defined by pitted erythrocyte count might reveal a parallel impairment of the immunological compartment ${ }^{[7]}$.

With increasing knowledge of the impairment of the immunological system in asplenic and hyposplenic states the trend is towards conserving the spleen in as many situations as possible. Partial splenectomy causes a transitory depression of the humoral immunity and enables an adequate phagocytic response. In patients who have undergone partial splenectomy for thalassemia, the risk of overwhelming post splenectomy sepsis (OPSI) is much lower.

Understanding the entities of splenosis and splenunculi (accessory spleen) is essential for evaluation of the immunological and haematological aftermath of splenectomy. Splenosis is a condition wherein fragments of a ruptured spleen are shed into the peritoneal cavity ${ }^{[8]}$. These pick up the blood supply from the area on which they are implanted and grow functionally. Splenotic nodules are living and functional splenic tissue. They are multiple in number, devoid of hilum, capsule and trabeculae. They derive blood supply from the omental vessels and their locations are altogether different from accessory spleens or splenunculi which are usually situated in vicinity of the splenopancreatic and gastro splenic ligaments ${ }^{[9]}$. The presence of splenosis is confirmed by the reduction in circulating pitted erythrocytes in splenectomised patients. However normal pitted erythrocytes counts are seen in patients after partial splenectomy or splenorrhaphy. Deliberate auto transplantation or spontaneous splenosis patients have pitted erythrocyte counts intermediate between normal counts and counts seen in splenectomised patients ${ }^{[10]}$

In patients who have splenosis following splenectomy, the concentration of pitted erythrocytes correlates with the mass of regenerated splenic tissue. The volume of replanted splenic tissue should be at least $30 \mathrm{cc}$ to ensure a fairly normal immunological outcome ${ }^{[11]}$. The antibody response after pneumococcal vaccination is satisfactory in patients who have splenosis. However splenosis does not guarantee full protection against OPSI. Possible factors that restrict the immunological efficacy of splenotic nodules are small size and poor blood supply which reduces the contact between the particulate antigens and splenic phagocytes.

\section{Hypo splenic states associated with medical conditions}

Functional hyposplenism is seen in $36 \%$ of HIV patients ${ }^{[12]}$. In patients suffering from AIDS the splenic function correlates well with the activity of the opsonising factor tuftsin derived from the spleen ${ }^{[13]}$. Untreated there is depletion of IgM memory B cells especially in patients with CD4 counts less than 300.

In graft versus host disease associated with bone marrow transplantation there is an increase in the count of pitted erythrocytes and Howell Jolly bodies suggestive of functional hyposplenism. Hyposlenism affects $15-40 \%$ of patients who have undergone allogenic bone marrow transplantation [14]. Decreased splenic size is associated with decrease in $\operatorname{IgM}$ memory cells. Such patients require lifelong antibiotic prophylaxis to prevent serious infectious complications.

Coeliac disease is associated with hyposplenism ${ }^{[15]}$. Coeliac disease is an immune mediated enteropathy induced in genetically susceptible individuals by the ingestion of gluten. Hyposplenism with or without splenic atrophy is seen in 33 to $76 \%$ of patients. The degree of hyposplenism correlates with the duration of pre exposure to gluten. Hyposplenism is seen in $19 \%$ of uncomplicated coeliac disease patients, 59\% with autoimmune disease and $80 \%$ with pre malignant or malignant complication ${ }^{[15]}$. Splenic atrophy and decreased IgM memory cells are associated with increased susceptibility to infection ${ }^{[16]}$. Sickle cell anaemia is associated with an enlarged spleen in the early years of life. This is due to vaso-occlusive congestion secondary to recurrent episodes of erythrocyte sickling. This leads to engorgement of sinusoids resulting in functional hyposplenism [17]. Such patients are prone to infection by encapsulated bacteria. Repeated splenic infarcts and consequent fibrosis lead to splenic atrophy (auto splenectomy) and finally irreversible hyposplenism [18]. Lifelong antibiotic prophylaxis and vaccination are necessary in these patients.

\section{Complications of splenectomy}

Splenectomy is performed for various haematological conditions. However trauma still continues to be a leading indication for splenectomy. Splenectomy is associated with a wide spectrum of technical and immunological complications. Aware ness of these complications is essential to adopt preventive measures in order to reduce the morbidity and mortality associated with splenectomy.

\section{Haemorrhage}

Haemorrhage is one of the most dreaded complications of splenectomy. It may either be primary or reactionary. Primary haemorrhage originates from various sources. Release of vascular adhesions, tear in the capsule while dissecting, injury to the splenic vessels, short gastric vessels and to the tail of the pancreas can cause torrential intraoperative bleeding [19] Reactionary haemorrhage usually arises from the small vessels in the posterior abdominal wall or the diaphragm. Bleeding is more severe in case of myeloproliferative disorders [19] Meticulous preoperative correction of coagulation defects, 
excellent visualisation of important structures, careful dissection and rapid control of bleeding will minimise the occurrence of intraoperative and postoperative bleeding. Post-operative bleeding usually manifests with tachycardia, hypotension, falling haematocrit and a sudden increase in bloody output in the drain. Prompt exploration is necessary. Evacuation of the haematoma with surgical control of bleeding is essentially the treatment. Anticipating coagulopathy as in massive transfusion and administering prophylactic blood products like fresh frozen plasma and cryoprecipitate can prevent DIC.

\section{Atelectasis}

Atelectasis can affect a significant number of patients undergoing splenectomy. It more commonly seen in elderly, overweight individuals as well as in chronic smokers and those suffering from COPD. It usually manifests in the first 48 hours after surgery as febrile episodes in majority of patients. The aetiology is a combination of obstructive and non-obstructive factors ${ }^{[19]}$. Secretions from COPD, intubation and the effect of anaesthetic agents play a significant role. The portion of the lung that is either dependent or severely compressed during the course of splenectomy, more so the lower lobe of the left lung is first to develop closure of bronchioles due to regional volume depletion. The clinical features are fever, tachycardia and breathlessness. Physical examination will reveal scattered rales, decreased breath sounds at the base. Monitoring of the oxygen saturation is essential in order to determine the need for supplemental oxygen or ventilator support. Early mobilisation, frequent change of posture, encouraging the patient to cough, use of an incentive spirometer and regular respiratory exercises can prevent this complication.

\section{Sub phrenic abscess}

This may occur as an isolated complication due to an infected haematoma or as a result of injury to an adjacent organ like the stomach or colon especially in patients with massively enlarged spleen ${ }^{[19,20]}$. Meticulous haemostasis during dissection of the left upper quadrant organs can significantly reduce the chances of developing an abscess. Placement of closed drain is helpful especially when a large dead space persists. Persistent intermittent spiking fever is the commonest feature. Chest $\mathrm{x}$ ray will reveal pleural effusion, raised left hemi diaphragm, basal infiltration and atelectasis. Abdominal x-ray will reveal ileus, air fluid levels, free gas pockets, effacement of the properitoneal or psoas outline and in a few cases even displacement of viscera. Ultrasound is helpful in diagnosis. However computed tomography (CT) is more reliable and confirmatory. At times the diagnosis may be difficult in areas with multiple thick walled bowel loops or if a pleural effusion overlies the sub phrenic abscess. Hence occasionally a large abscess could be missed. CT has an additional advantage of enabling an aspiration. CT guided aspiration enables differentiation between sterile and infected collection. Percutaneous drainage can also be done under CT guidance. Percutaneous drainage is the preferred method for a single well localized, superficial abscess which does not have a fistulous communication nor does it contain any particulate debris ${ }^{[19]}$. CT guided insertion of a large catheter may be indicated in large abscesses. The success rate is $80 \%$ in simple abscesses and less than $50 \%$ in complex ones. Open drainage is indicated when the abscess fails to resolve or is very big in size with lot of particulate debris. Extra peritoneal approach with resection of $12^{\text {th }}$ rib provides wide access to the abscess cavity. The infected area is opened and all infected tissue debris removed. All loculations are broken down and a drain is placed in the cavity. The drain is kept till the infection resolves ${ }^{[19]}$. The choice of antibiotics is determined by culture and sensitivity report of the pus.

\section{Thrombocytosis}

Post splenectomy thrombocytosis peaks in 7 to 10 days ${ }^{[19]}$. It rarely returns to normal. The circulating platelets exceed $10^{6}$ cells $/ \mathrm{ml}$. Thromboembolic complications do develop as a result of thrombocytosis but the occurrence of such complications does not correlate with the degree of thrombocytosis. Preventive measures for complications include elevation of the legs, graduated pressure compression stockings, aspirin or low molecular heparin therapy. The risk of deep vein thrombosis (DVT) is extremely high after splenectomy. Hence routine DVT prophylaxis is essential after splenectomy. Monitoring of platelet count for heparin induced thrombocytopenia (HIT) is essential as this peaks in 5-10 days. Portal vein thrombosis is another complication $[19,20,21]$. The patient may present with fever, abdominal pain, loose motions and deranged liver function tests. Systemic antibiotics and anticoagulation is the mainstay of treatment.

\section{Gastric complications}

The greater curvature of the stomach can be damaged while mobilising the spleen ${ }^{[20]}$. The short gastric vessels may rupture or ligatures could be tied too close to the stomach wall thereby causing damage ${ }^{[20]}$. This could either lead to acute dilatation of the stomach or greater curve necrosis. A prophylactic nasogastric tube can prevent acute dilatation. It can also help in early diagnosis of greater curve necrosis which can manifest as hematemesis accompanied with abdominal signs.

\section{Pancreatic complications}

Pancreas is the organ which is in close vicinity to the spleen. Injury to the tail of pancreas is commonly encountered, especially while dissecting the hilar region of the spleen. For a moderately damaged pancreas, control of bleeding, ligation of exposed ducts and closed drainage are sufficient. However if there is extensive damage then resection of the tail of pancreas and closed drainage of the region is essential. Unrecognized pancreatic injury may manifest as pancreatitis, pseudocyst or a fistula. The best preventive surgical practice is to keep closed suction drainage for 4 to 5 days and administer prophylactic octreotide.

Acute pancreatitis is seen in significant number of patients who have undergone splenectomy ${ }^{[19]}$. However the disease takes a mild course with minimal organ dysfunction. Pain management, fluid replacement, nutritional support either TPN or oral are therapeutic. Pancreatic infection may develop in a few cases not managed adequately in the initial phase necessitating antibiotic therapy.

Peripancreatic fluid collection is usually due to pancreatic duct disruption [19]. Majority of cases will have a self-remitting course. When clinical symptoms persist beyond one week, further investigation and treatment is warranted. CT or MRCP will reveal the presence of duct disruption with a pseudocyst formation. An ERCP could be done in order to stent the duct. In a few cases external drainage is essential.

Post splenectomy necrotising pancreatitis may be seen in a few cases ${ }^{[19]}$.

A contrast enhanced CT will reveal necrosis revealed by lack of vascular perfusion and the presence of necrotic pancreatic tissue debris. The treatment of sterile necrosis is conservative. However if the quantum of necrosis exceeds $50 \%$ with 
superadded infection then a surgical necrosectomy is essential. If untreated the mortality of necrotising pancreatitis is $50-80 \%$. However a timely necrosectomy can reduce the mortality to 10 $40 \%$.

Pancreatic fistula following splenectomy is a cumbersome complication to treat $[19,20,21,22]$. The pancreatic duct or one of its major branches is disrupted either by direct trauma at the time of surgery or by the severity of post pancreatitis inflammatory process. The mortality is $8-10 \%$ due to sepsis or haemorrhage. A pancreatic fistula usually presents on the third or fourth postoperative day. It is heralded by a sudden increase in drain output of serous or cloudy, non-bilious fluid which usually has a high amylase concentration. Intraoperative placement of a tube drain helps in early diagnosis ${ }^{[23]}$. However if no drain was placed initially then the presentation becomes elusive. There could be spontaneous drainage through the surgical wound or intraoperative collection with abdominal signs. An MRCP is extremely helpful in the initial evaluation. The site of the duct disruption is identified. Associated fluid collections are also located. Once confirmed the output of the fistula has to be estimated. If it is a low output fistula with output less than 200 $\mathrm{ml}$ per day then conservative treatment will suffice. However if it exceeds $200 \mathrm{ml}$ per day then that is worrisome. It suggests either a major pancreatic ductal disruption or some ductal anomaly. The therapeutic regimen comprises of daily measurement of the fistula output, correction of fluid and electrolyte abnormalities, treating acidosis, systemic antibiotics, TPN and managing skin complications. Most fistulas will close by this treatment. Administering octreotide is of great help and hastens fistula closure and thereby the cost of hospitalisation. Surgical intervention is indicated if the fistula arises from ductal disruption at the level of the body of the pancreas with persistently high output. A distal pancreatectomy is indicated in such cases. However the morbidity and mortality associated with procedure is quite high.

\section{Overwhelming post splenectomy infection (OPSI)}

Overwhelming post splenectomy infection (OPSI) is a rare condition with high mortality following splenectomy ${ }^{[24]}$. The condition is preventable if proper precautions are taken. But once developed, it follows a fulminant course with extremely high mortality. Hence aware ness of the presenting features is essential.

Risk factors for developing OPSI are age at which splenectomy was done, subsequent time interval from the surgery, indication for splenectomy and the overall immunological status of the patient ${ }^{[24,25]}$. Children are at a higher risk of developing OPSI after splenectomy especially in the first two years after surgery than adults. Patients who undergo splenectomy for haematological disorders have higher risk of developing OPSI than those who have undergone splenectomy for trauma. Patients with autoimmune disorders or those who have received chemotherapy have a weakened immune system. These individuals have a high risk of developing OPSI by virtue of weakened immune defence systems.

The liver Kupffer cells clear most of the opsonized bacteria. However encapsulated organisms who resist antibody binding are cleared by the spleen. Encapsulated organisms such as streptococcus pneumoniae are resistant to phagocytosis but are quickly controlled by type specific antibody which is produced by the spleen. Prompt production of antibodies to new stimuli is absent in a splenectomised individual thereby rendering the subject vulnerable to a wide spectrum of organisms with special mention of encapsulated organisms such as streptococcus pneumoniae, N. meningitides, H. influenza and S. pyogenes in descending order of frequency ${ }^{[25]}$.

OPSI presents with cryptic infection. The symptoms are fever, shivering, myalgia, vomiting, loose motions and headache. This then leads to septic shock with development of anuria, hypotension and hypoglycaemia. Pneumonia and meningitis are common accompaniments. The later clinical course may lead to Waterhouse-Frederickson syndrome due to bilateral adrenal haemorrhage. The postulated mechanisms are loss of splenic phagocytic function, decreased serum immunoglobulin levels, suppression of lymphocyte activity and decrease in the production of opsonins. Severe septic shock, respiratory distress and DIC cause multiorgan failure and death. Intravenous fluids, antibiotics, ionotropes, packed cells, fresh frozen plasma, cryoprecipitates and ventilatory support constitute the mainstay of treatment. Despite aggressive treatment the mortality is 50$70 \%$ within the first 24 hours ${ }^{[25,26]}$.

Awareness of preventive measures for OPSI is of utmost importance. Preventive strategies begin with patient education [27]. Patients who have undergone splenectomy should be informed about the high incidence of this lethal complication and the presenting features of this condition. Individuals travelling to endemic regions of malaria should be advised chloroquine prophylaxis. Any individual who has a dog bite, animal bite or is undergoing any dental procedure should be adequately treated with antibiotics.

Children younger than five years should be started on prophylactic antibiotics. This significantly reduces the chance of developing OPSI. In adults amoxicillin or phenoxymethyl penicillin is recommended. Long term prophylaxis is recommended for patients suffering from haematological conditions or who have very poor immunity ${ }^{[27]}$.

Vaccination is mandatory for patients undergoing splenectomy. Polyvalent pneumococcal vaccination should be done two weeks prior to surgery ${ }^{[28]}$. In emergency splenectomies for trauma, vaccination is done immediately after surgery during the early convalescent period ${ }^{[29]}$. In addition immunisation against $\mathrm{H}$ influenza and $\mathrm{N}$ meningitides is also recommended. Revaccination is essential if the antibody titres are inadequate [29].

\section{Conclusion}

Post splenectomy and hyposplenic states predispose patients to a wide spectrum of complications ranging from surgical to serious immunological and septic complications. Majority can be prevented by meticulous preparation, safe surgical technique and post procedure infection prophylaxis. Adequate patient education, antibiotic prophylaxis and vaccination can significantly reduce the incidence of OPSI.

\section{Acknowledgement}

The author would like to thank Mr. Parth Vagholkar for his help in typesetting the manuscript.

\section{References}

1. Mebius RE, Kraal G. Structure and function of the spleen. Nat Immunol. 2005; 5:606-16.

2. William BM, Thawani N, Sae-Tia S, Corazza GR. Hyposplenism: a comprehensive review. Part II. Clinical manifestations, diagnosis and management. Hematology. 2007; 12:89-98.

3. William BM, Corazza GR. Hyposplenism: a comprehensive review. Part I. Basic concepts and causes. Hematology. 2007; 12:1-13. 
4. Sills RH. Splenic function: physiology and splenic hypofunction. Crit Rev Oncol Hematol. 1987; 7:1-36.

5. Spencer RP, Gupta SM. The spleen: diagnosis of splenic diseases using radiolabeled tracers. Crit Rev Lab Sci. 1989; 27:299-318.

6. Dhawan V, Spencer RP, Pearson HA, Sziklas JJ. Functional asplenia in the absence of circulating Howell-Jolly bodies. Clin Nucl Med. 1977; 2:395-96.

7. Holroyde CP, Oski FA, Gardner FH. The pocked erythrocyte: Red-cell surface alterations in reticuloendothelial immaturity of the neonate. N Engl J Med 1969; 281:516-20.

8. Timens W, Leemans R. Splenic autotransplantation and the immune system. Adequate testing required for evaluation of effect. Ann Surg. 1992; 215:256-60.

9. Fremont RD, Rice TW. Splenosis: a review. South Med J. 2007; 100:589-93.

10. Buchbinder JH, Lipkoff CJ. Splenosis: multiple peritoneal splenic implant following abdominal injury: a report of a case and review of the literature. Surgery. 1939; 6:927-34.

11. Corazza GR, Tarozzi C, Vaira D, Frisoni M, Gasbarrini G. Return of splenic function after splenectomy: how much tissue is needed? BMJ. 1984; 289:861-64.

12. Grotto HZ, Costa FF. Hyposplenism in AIDS. AIDS. 1991; 5:1538-40.

13. Corazza GR, Zoli G, Ginaldi L et al. Tuftsin defi ciency in AIDS. Lancet. 1991; 337:12-13.

14. Kalhs P, Panzer S, Kletter K et al. Functional asplenia after bone marrow transplantation. A late complication related to extensive chronic graft-versus-host disease. Ann Intern Med. 1988; 109:461-64.

15. Corazza GR, Zoli G, Di Sabatino A, Ciccocioppo R, Gasbarrini G. A reassessment of splenic hypofunction in celiac disease. Am J Gastroenterol. 1999; 94:391-97.

16. Ludvigsson JF, Olen O, Bell M, Ekbom A, Montgomery SM. Coeliac disease and the risk of sepsis. Gut. 2008; 57:1074-80.

17. Pearson HA, McIntosh S, Ritchey AK. Developmental aspects of splenic function in sickle cell anemia. Blood. 1979; 53:358-65.

18. Pearson HA, Spencer RP, Cornelius A. Functional asplenia in sickle-cell anemia. N Engl J Med. 1969; 281:923-26.

19. Shatney $\mathrm{CH}$. Complications of splenectomy. Acta Anaesthesiol Belg. 1987; 38:333-339.

20. Stallard S, Mc Pherson SG. Gastric necrosis and perforation following splenectomy for massive splenomegaly. Scot Med J. 1990; 35:86.

21. Winslow ER, Brunt LM, Drebin JA, Soper NJ, Klingensmith ME, Dunn E. Portal vein thrombosis after splenectomy. The American Journal of Surgery. 2002; 184:631-635.

22. Cohn LH. Local infections after splenectomy. Arch Surg. $1965 ; 23: 90$.

23. Daoud FS, Fisher DC, Hafner CD. Complication following splenectomy with special emphasis on drainage. Arch Surg. 1966; 90:32.

24. Brigden ML, Pattullo AL. Prevention and management of overwhelming postsplenectomy infection--an update. Crit Care Med. 1999; 27:836-842.

25. Di Cataldo A, Puleo S, Li Destri G, Racalbuto A, Trombatore G, Latteri $\mathrm{F}$ et al. Splenic trauma and overwhelming postsplenectomy infection. Br J Surg. 1987; 74:343-345.

26. Davies JM, Barnes R, Milligan D. Update of guidelines for the prevention and treatment of infection in patients with an absent or dysfunctional spleen. Clin Med. 2002; 2:440-443.

27. Williams DN, Kaur B. Postsplenectomy care. Strategies to decrease the risk of infection. Postgrad Med. 1996; 100:195-198.

28. Shatz DV. Vaccination considerations in the asplenic patient. Expert Rev Vaccines. 2005; 4:27-34.

29. Shatz DV, Romero-Steiner S, Elie CM, Holder PF, Carlone GM. Antibody responses in postsplenectomy trauma patients receiving the 23-valent pneumococcal polysaccharide vaccine at 14 versus 28 days postoperatively. J Trauma. 2002; 53:1037-42. 